\title{
APLICAÇÃO PREVENTIVA E CURATIVA DE FUNGICIDAS PARA CONTROLE DA HELMINTOSPORIOSE EM MILHO
}

\author{
J.N.CAMERA ${ }^{1}$, J.KOEFENDER ${ }^{2}$, D.P. GOLLE ${ }^{3}$, E.F.FLORES ${ }^{4}$, R.P. BORTOLOTTO ${ }^{5}$, A. SCHOFFEL ${ }^{6}$, C.C. DEUNER ${ }^{7}$ \\ Universidade de Cruz Alta ${ }^{1,2,3,5}$, Universidade de Santa Maria ${ }^{6}$, Universidade de Passo Fundo ${ }^{7}$ \\ ju_camera@yahoo.com.br ${ }^{1}$
}

Artigo submetido em 23/10/2017 e aceito em 09/04/2019

DOI: $10.15628 /$ holos.2019.6467

\begin{abstract}
RESUMO
O controle químico das doenças é uma das medidas de controle mais utilizadas, principalmente quando se objetiva o controle rápido e preciso. $\mathrm{O}$ objetivo deste trabalho foi verificar o comportamento de diferentes grupos químicos de fungicidas quando aplicados de forma preventiva e curativa visando controle da helmintosporiose do milho. Os fungicidas utilizados foram propiconazol, azoxistrobina, ciproconazol + azoxistrobina e benzovindiflupir + azoxistrobina $e$ protioconazol + trifloxistrobina. O híbrido utilizado foi Pioneer $\mathrm{P} 1630 \mathrm{H}$ suscetível à Exserohilum turcicum. Utilizou-se uma suspensão $20 \times 103$ esporos $\mathrm{mL}^{-1}$, as plantas foram inoculadas por aspersão no estádio V4 (quarta folha expandida, apresentando colar, lígula e aurícula visíveis). $O$ delineamento experimental foi blocos
\end{abstract}

casualizados, com três repetições. No experimento preventivo as plantas foram inoculadas nos dias $1,4,8$, $10,16,19$ e 22 . No controle curativo as aplicações de fungicidas foram nos dias 1, 4, 6, 11, 14, 17 e 20. Avaliouse a severidade (\%), eficiência de controle e área da lesão. Os fungicidas protioconazol + trifloxistrobina, ciproconazol + azoxistrobina e azoxistrobina mostraramse eficientes por até 21 dias após a inoculação de $E$. turcicum. Os fungicidas propiconazol e benzovindiflupir + azoxistrobina foram eficientes para o controle de $E$. turcicum. O fungicida protioconazol + trifloxistrobina apresentou a menor taxa de expansão de lesão de $E$. turcicum quando aplicado curativamente enquanto que $o$ fungicida benzovindiflupir + azoxistrobina apresentou a maior taxa de expansão de lesão de E. turcicum.

PALAVRAS-CHAVE: Exserohilum turcicum, controle preventivo, controle curativo.

\section{PREVENTIVE AND CURATIVE APPLICATION OF FUNGICIDES FOR THE CONTROL OF HELMINTOSPORIOSIS IN CORN}

\begin{abstract}
The chemical control of diseases is one of the most used control measures, especially when aiming for fast and precise control. The objective of this work was to verify the behavior of different chemical groups of fungicides when applied in a preventive and curative way aiming the control of corn helmintosporiosis. The fungicides used were propiconazole, azoxystrobin, cyproconazole + azoxystrobin and benzovindiflupir + azoxystrobin and prothioconazole + trifloxystrobin. The hybrid used was Pioneer $\mathrm{P} 1630 \mathrm{H}$ susceptible to Exserohilum turcicum. The suspension $20 \times 10^{3}$ spore $\mathrm{mL}^{-1}$ was used, the plants were inoculated by $\mathrm{V} 4$ stage spraying (fourth expanded leaf, showing visible collar, ligule and auricle). The experimental design was randomized blocks with three replicates. In the preventive experiment the plants were
\end{abstract}

inoculated on days $1,4,8,10,16,19$ and 22 . In the curative control the fungicide applications were on days $1,4,6,11,14,17$ and 20 . It was evaluated The severity (\%), the control efficiency and the area of the lesion. The fungicides prothioconazole + trifloxystrobin, cyproconazole + azoxystrobin and azoxystrobin were efficient for up to 21 days after inoculation of E. turcicum. The fungicides propiconazole and benzovindiflupir + azoxystrobin were efficient for the control of E. turcicum. The fungicide prothioconazole + trifloxystrobin presented the lowest rate of lesion expansion of E. turcicum when applied curatively while the fungicide benzovindiflupir + azoxystrobin presented the highest rate of lesion expansion of $E$. turcicum.

KEYWORDS: Exserohilum turcicum, control preventive, control curative. 


\section{INTRODUÇÃO}

A cultura do milho (Zea mays L.) apresenta grande importância, sendo cultivada em todas as partes do mundo, de acordo com os dados da USDA (2017), a produção mundial de milho encontrase, hoje, por volta de 1.011,07 bilhão de toneladas, sendo que $36,25 \%$ provêm dos EUA, o Brasil que ocupa terceiro lugar com média de produção atual em torno de 97 milhões de toneladas (CONAB, 2017).

As doenças foliares causam sérios danos, prejudicando a cultura, reduzindo a área fotossintética e inibindo a translocação de assimilados desde a sua fonte de produção até as áreas de crescimento e deposição de material de rendimento (Gomes et al., 2011). Dentre as doenças que ocorrem no milho destaca-se a helmintosporiose, causada pelo fungo Exserohilum turcicum (Pass.) Leonar \& Suggs., que é uma das doenças mais antigas e importantes relacionadas à cultura do milho no Brasil (Alvim et al., 2010).

Em epidemias de $E$. turcicum, a incidência em plantas pode chegar a $100 \%$, causando grandes reduções no rendimento (Raymundo \& Hooker, 1981). As epidemias ocorrem mais freqüentemente nas regiões sul e oeste do Brasil, onde causam danos severos no rendimento de grãos (Esteves, 1989), chegando até 50\% (Raymundo \& Hooker, 1981).

Os sintomas da helmintosporiose do milho podem ser observados uma semana após a inoculação do patógeno nas folhas (Elliot \& Jenkins, 1946), como lesões foliares de formato elíptico e alongado variando o comprimento de 2,5-15,0 cm, predominantemente de cor cinza, às vezes verde-acinzentadas ou pardas, sem bordos delimitados de coloração parda-avermelhada.

O controle químico das manchas foliares na cultura do milho tem demonstrado bons resultados de controle e manutenção de produtividade quando aplicados no momento correto (Lago \& Nunes, 2008). Esses resultados são visualizados, normalmente, como incremento de produtividade em relação a áreas não pulverizadas (Pinto et al., 2004).

Os fungicidas apresentam ação protetora, curativa erradicante, e anti-esporulante. A ação protetora se expressa quando esse é aplicado antes do patógeno infectar os tecidos da planta. Ação curativa consiste na capacidade do fungicida em limitar o desenvolvimento do patógeno no interior dos tecidos, quando aplicado no período latente, ou seja, no intervalo entre a penetração e o aparecimento dos primeiros sintomas. Entre os fatores que podem influenciar a ação curativa de um fungicida destacam-se a suscetibilidade do hospedeiro, a pressão de doença, as condições meteorológicas e o momento da aplicação do fungicida na planta (Genet et al., 2000).

O objetivo deste trabalho foi verificar o comportamento de diferentes grupos químicos de fungicidas quando aplicados de forma preventiva e curativa visando controle da helmintosporiose do milho.

\section{MATERIAL E MÉTODOS}

Os experimentos foram conduzidos no Laboratório de Fitopatologia e casa-de-vegetação da Faculdade de Agronomia e Medicina Veterinária da Universidade de Passo Fundo. O delineamento 
experimental de todos os ensaios foi em blocos ao acaso com três repetições, sendo que cada repetição foi composta por um vaso com três plantas. Foram realizadas aplicações preventivas e curativas de fungicidas.

\section{Aplicação preventiva de fungicidas em milho}

Para a realização do experimento, utilizou-se o híbrido de milho Pioneer $\mathrm{P} 1630 \mathrm{H}$, suscetível a helmintosporiose. A inoculação utilizou-se uma suspensão $20 \times 103$ esporos $\mathrm{mL}^{-1}$, as plantas foram inoculadas por aspersão no estádio V4 (quarta folha expandida, apresentan do colar, lígula e aurícula visíveis). $\mathrm{O}$ inóculo foi multiplicado no hibrido Pioneer $\mathrm{P} 1630 \mathrm{H}$ e mantidos em casa de vegetação, sendo que para o preparo da suspensão foram coletadas folhas com intensa esporulação e colocadas em recipiente para agitação com água e espalhante polioxietilenosorbitano (Tween 20) para a liberação dos esporos. Em seguida, procedeu-se a quantificação dos esporos em câmara de 'Neubauer' (Alfenas \& Mafia, 2007), sendo a suspensão calibrada para 20x103 esporos mL-1.

As plantas foram inoculadas nos dias 1, 4, 8, 10, 16, 19 e 22 após a aplicação dos fungicidas (Tabela 1). Decorrido trinta dias, procedeu-se a avaliação de severidade da doença e medição da área da lesão.

Tabela 1: Fungicidas utilizados para o controle de Exserohilum turcicum em milho. UPF, Passo Fundo/RS, 2015

\begin{tabular}{|c|c|c|c|}
\hline \multirow{2}{*}{$\begin{array}{c}\text { Nome } \\
\text { comercial }\end{array}$} & \multirow{2}{*}{ Ingrediente ativo } & \multicolumn{2}{|c|}{ Dose $^{34}$} \\
\hline & & (g i.a ha-1) & (L ou kg p.c ha-1) \\
\hline Tilt & Propiconazol & 100 & 0,4 \\
\hline Priori & Azoxistrobina & 75 & 0,3 \\
\hline Priori Xtra ${ }^{1}$ & Ciproconazol + azoxistrobina & $24+60$ & 0,3 \\
\hline Elatus ${ }^{1}$ & Benzovindiflupir + azoxistrobina & $30+60$ & 0,2 \\
\hline Fox ${ }^{2}$ & Protioconazol + trifloxistrobina & $70+60$ & 0,4 \\
\hline
\end{tabular}

${ }^{1}$ Adicionado Nimbus $600 \mathrm{~mL} \mathrm{ha}{ }^{-1} ;{ }^{2}$ Adicionado Aureo $0,25 \% ;{ }^{3}$ Ingrediente ativo. ${ }^{4}$ Produto comercial.

Para a severidade, estimou-se a área foliar atacada pela doença, através da severidade estimada. Com base nos dados da severidade, calculou-se a eficiência de controle utilizando-se a fórmula de Abbot (1925):

$$
\text { Eficiência }(E \%)=\left(\frac{\text { severidade da testemunha }- \text { severidade da parcela tratata }}{\text { severidade da testemunha }}\right) * 100
$$

Para área das lesões, primeiramente, as lesões foram marcadas e numeradas com marcador permanente, em seguida, as mesmas foram mensuradas com auxílio de um paquímetro digital (precisão 0,01 mm) com intervalo de dois ou três dias, totalizando cinco avaliações. Como as lesões não são retangulares utilizou-se um fator de correção $(0,76)$ obtido anteriormente através da comparação da área real da lesão com as medições de largura e comprimento. Com os dados obtidos das avaliações dos 22 dias após a aplicação dos fungicidas procedeu-se a análise estatística, as variáveis com teste $F$ significativo foram submetidas ao teste de comparação de médias, de Tukey, a 5\% de probabilidade, utilizou-se o software Assistat. 


\section{Aplicação curativa de fungicidas em milho}

Utilizou-se neste experimento o híbrido Pioneer $\mathrm{P} 1630 \mathrm{H}$, suscetível a helmintosporiose. As plantas de milho foram semeadas em vaso com $10 \mathrm{~kg}$ de solo hortado, sendo o experimento foi mantido em casa-de-vegetação com fotoperíodo de 12 horas. Quando as plantas atingiram o estádio V4, foram pulverizadas com fungicidas (Tabela 1) nos dias 1, 4, 6, 11, 14, 17 e 20 após a inoculação das plantas com o fungo, utilizando pulverizador costal pressurizado $\operatorname{com} \mathrm{CO}_{2}$, calibrado para volumes de calda de $100 \mathrm{~L} \mathrm{ha}^{-1}$.

A multiplicação do inóculo, inoculação e incubação das plantas e avaliações foram realizadas da mesma maneira que para o experimento com aplicação preventiva com esse mesmo fungo. Primeiramente, procedeu-se a inoculação do fungo e em seguida a aplicação dos fungicidas. Decorrido trinta dias, procedeu-se a avaliação da mesma maneira para o experimento com aplicação preventiva de fungicidas para controle desse fungo.

Com os dados obtidos na avaliação de 4 dias após a inoculação do fungo, fez-se a taxa de expansão da lesão (avaliou-se aos 1, 3, 6, 8 e 10 dias) a fim de observar a taxa de expansão da lesão diária para os diferentes fungicidas testados.

\section{RESULTADOS E DISCUSSÃO}

\section{Aplicação preventiva de fungicidas em milho}

Aos 21 dias após a aplicação dos fungicidas, observou-se que para a severidade de helmintosporiose, os fungicidas protioconazol + trifloxistrobina, propiconazol e ciproconazol + azoxistrobina, apresentaram o menor valor dessa variável e controle de 99,63\%, 94,41\% e 93,53\% respectivamente. Dados semelhantes foram obtidos para número de lesões/folha e área da lesão, sendo que a eficiência de controle para esta variável foi de 98,82\%, 92,92\% e 91,10\% e para área da lesão 72,29\%, 70,03\% e 67,72 \% (Tabela 2).

\section{Aplicação curativa de fungicidas}

Tanto para severidade como para área da lesão, verificou-se que todos os fungicidas apresentam controle satisfatório até 15 dias após a inoculação de E. turcicum (Figura 1).

Com os dados obtidos na avaliação aos quatro dias após a inoculação do fungo, fez-se a taxa de expansão da lesão, a maior taxa de expansão da lesão para os tratamentos com fungicidas foi de $96,21 \mathrm{~mm}^{2}$ ao dia para o benzovindiflupir + azoxistrobina, seguido dos fungicidas azoxistrobina $\left(47,18 \mathrm{~mm}^{2}\right)$, propiconazol $\left(3,4 \mathrm{~mm}^{2}\right)$ e ciproconazol + azoxistrobina $\left(2,0 \mathrm{~mm}^{2}\right)$. A menor taxa de expansão da lesão foi obtida para o fungicida protioconazol + trifloxistrobina $\left(0,45 \mathrm{~mm}^{2}\right)$. A testemunha apresentou taxa de expansão de $203,41 \mathrm{~mm}^{2}$ ao dia (Figura 2). 
Tabela 2: Severidade estimada, número de lesões/folha e área da lesão 21 dias após a aplicação preventiva dos fungicidas visando controle de Exserohilum turcicum. UPF, Passo Fundo/RS, 2015

\begin{tabular}{|c|c|c|c|c|c|c|}
\hline Fungicidas & $\begin{array}{c}\text { Severidade } \\
\text { (\%) }\end{array}$ & $\begin{array}{c}\text { Controle } \\
(\%)\end{array}$ & $\begin{array}{l}\text { Número de } \\
\text { lesões/folha }\end{array}$ & $\begin{array}{c}\text { Controle } \\
(\%)\end{array}$ & $\begin{array}{c}\text { Área da } \\
\text { lesão }\left(\mathrm{mm}^{2}\right)\end{array}$ & $\begin{array}{c}\text { Controle } \\
\text { (\%) }\end{array}$ \\
\hline Testemunha & $38,65 a^{1}$ & - & $9,33 \mathrm{a}$ & - & $232,48 \mathrm{a}$ & - \\
\hline Propiconazol & $2,16 \mathrm{c}$ & 94,41 & $0,66 \mathrm{c}$ & 92,92 & $67,67 c$ & 70,03 \\
\hline $\begin{array}{l}\text { Benzovindiflupir } \\
+ \text { azoxistrobina }\end{array}$ & $12,16 \mathrm{~b}$ & 68,53 & $4,76 \mathrm{~b}$ & 48,98 & $134,43 b$ & 42,17 \\
\hline Azoxistrobina & $17,33 \mathrm{~b}$ & 55,16 & $3,67 \mathrm{~b}$ & 60,66 & $170,27 b$ & 26,75 \\
\hline $\begin{array}{l}\text { Ciproconazol + } \\
\text { azoxistrobina }\end{array}$ & $2,50 \mathrm{c}$ & 93,53 & $0,83 c$ & 91,1 & $75,03 \mathrm{c}$ & 67,72 \\
\hline $\begin{array}{l}\text { Protioconazol + } \\
\text { trifloxistrobina }\end{array}$ & $0,14 c$ & 99,63 & $0,11 c$ & 98,82 & $64,43 c$ & 72,29 \\
\hline CV (\%) & 15,34 & & 6,21 & & 9,34 & \\
\hline
\end{tabular}

${ }^{1}$ Médias seguidas de mesma letra na coluna não diferem ao nível de 5\% de probabilidade, pelo teste de Tukey.

O controle químico é um dos principais métodos de controle de doenças de plantas, quer pela facilidade de uso como também pelos resultados obtidos. Porém seu uso constante e sem critérios pode promover a seleção de fungos resistentes, colocando em risco a eficiência do método. Isso é indesejável à indústria química, aos produtores e à sociedade consumidora, conforme abordado por Ghini \& Kimati (2000).

Os fungicidas do grupo triazol, denominados inibidores da desmetilação (IDM) agem sobre o patógeno na inibição da reação de desmetilação do C-14 (Linhares \& Ghini, 2001). As estrobilurinas interferem na respiração mitocondrial, bloqueando a transferência de elétrons pelo complexo citocrômico bc1, através da inibição do óxido-redutase de ubihidroquinonacitocromo c (Ghini \& Kimati, 2000). Os citocromos são ferroproteínas que atuam sequencialmente, transferindo elétrons do $\mathrm{CoQH} 2$ para o oxigênio molecular. A interferência exercida pelo fungicida impede a liberação de energia e a formação de ATP (Forcelini et al., 2001).

A carboxamida possui elevada sistemicidade e grande espectro de ação em ascomicetos, basidiomicetos e deuteromicetos (mitospóricos), acrescentando consistência no residual de controle. Além disso, apresentam ação protetora e curativa, controlando inclusive patógenos resistentes a estrobilurinas. Estas indicam possível efeito na biossíntese de proteínas, lipídeos, DNA e RNA, além de ser observada maior transformação de glicose ou acetato em succinato e uma diminuição na transformação de citrato, malato e fumarato. A utilização de misturas com fungicidas de modo de ação diferentes aumenta o espectro de controle de fungos, o período residual das aplicações e contribuem para evitar o desenvolvimento da redução da sensibilidade de fungos aos fungicidas (Reis et al., 2010). 

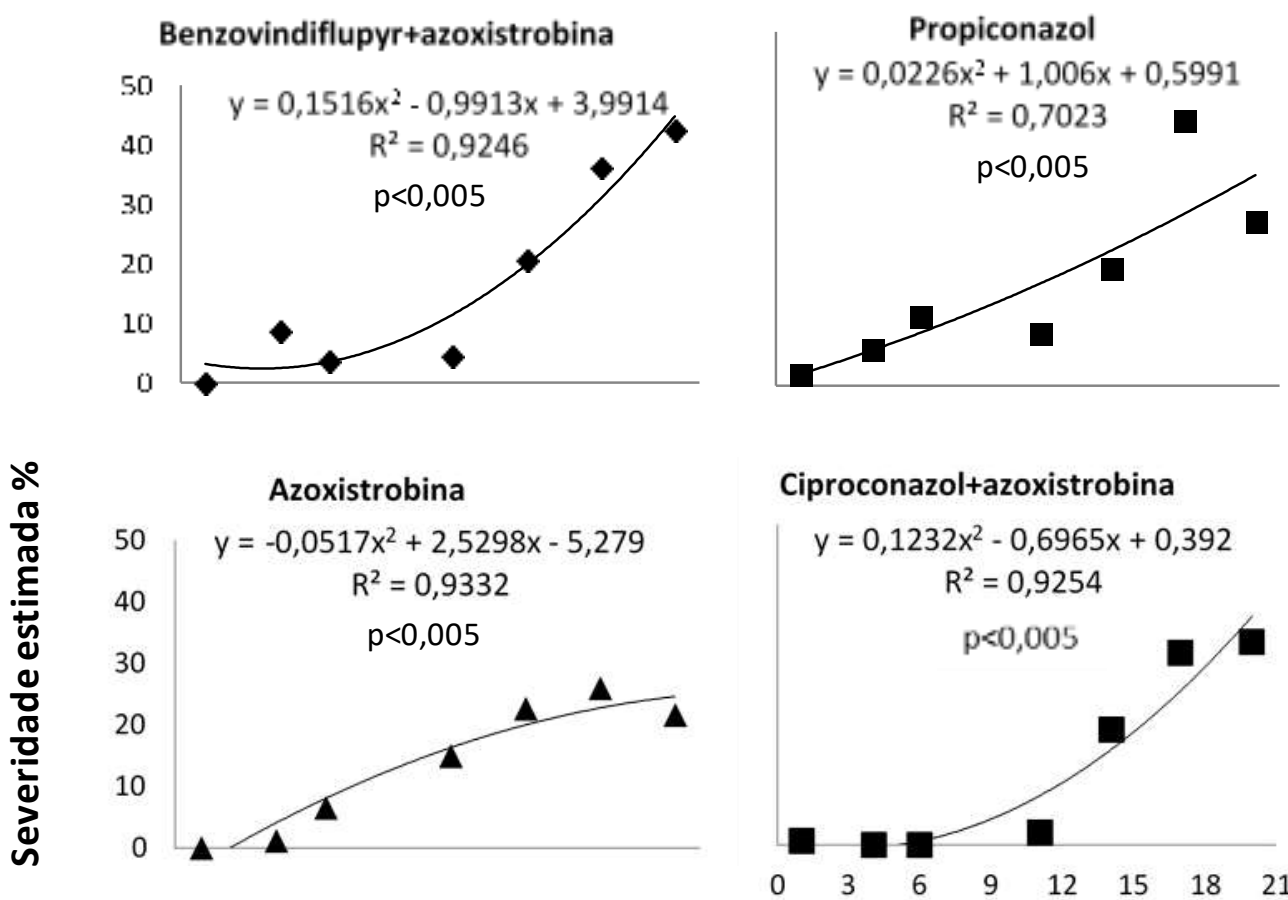

Protioconazol+trifloxistrobina

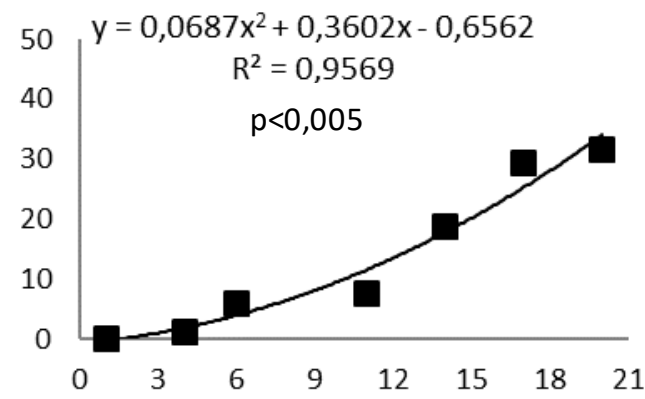

Dias após a inoculação

Figura 1: Severidade estimada (\%) no intervalo em dias entre a inoculação de Exserohilum turcicum em milho e a aplicação de fungicidas.

Segundo Boller et al. (2007), a eficácia do controle depende da idade da infecção. Uma aplicação de fungicida sobre infecções recém estabelecidas resulta na morte do patógeno. Azevedo (2007) relata o efeito curativo/erradicante mais pronunciado dos fungicidas até 48 a 72 horas após a infecção do patógeno. Em infeç̧ões mais antigas (maiores que meio período latente), a energia não mais utilizada para o crescimento é realocada para a reprodução, originando lesões e formando esporos, viáveis ou não. Esse processo perdura por três a quatro dias, às vezes mais se a temperatura estiver baixa. À primeira vista, esse fato surpreende o produtor, que percebe mais lesões após a aplicação do fungicida e pode interpretar como falha no controle. A causa, no entanto, está no elevado número de infecções em estágio de incubação, não visíveis, o que reforça a necessidade de se aplicar antecipadamente, preventivamente ou, no máximo quando do aparecimento dos primeiros sintomas (Boller et al., 2007). 


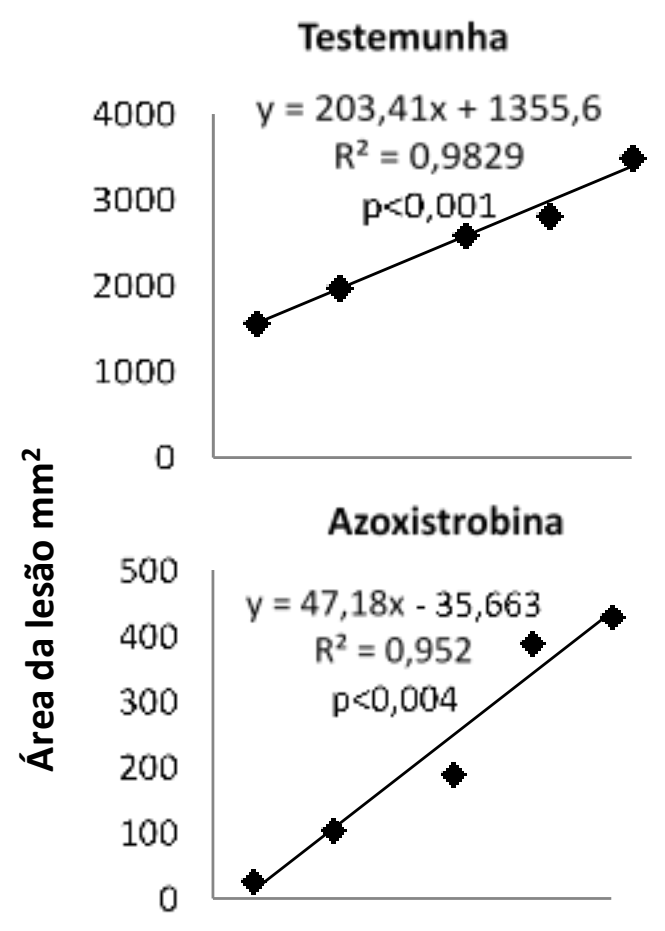

Ciproconazol+azoxistrobina

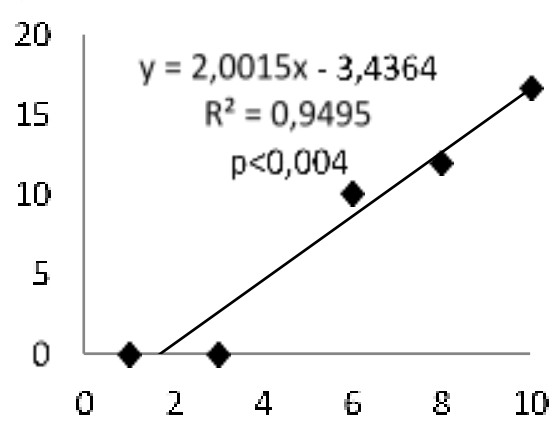

\section{Benzovindiflupyr+azoxistrobina}
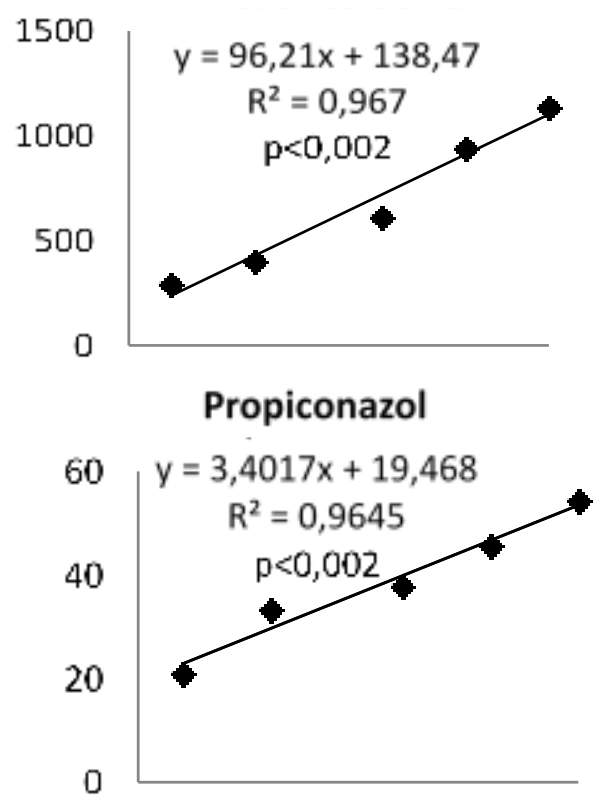

Protioconazol+trifloxistrobina

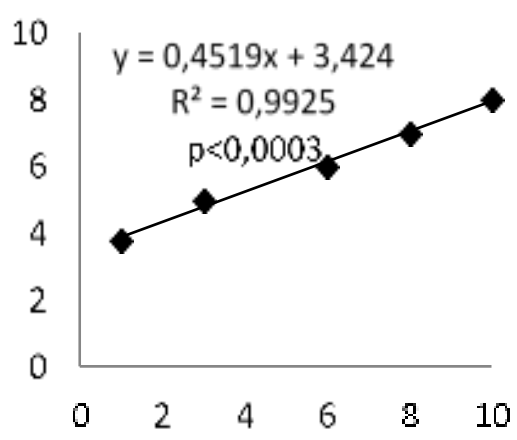

Dias após a inoculação

Figura 2: Aumento da lesão de helmintosporiose com aplicações de diferentes fungicidas, no período de 10 dias após a inoculação de Exserohilum turcicum. UPF, Passo Fundo, 2015.

Trabalhos realizados por Pinto (2004) mostram que para o controle de E. turcicum o fungicida que apresentou melhor controle foi tebuconazol (200 g i.a. ha-1 $)$. A eficiência de tebuconazol no controle dessa doença também foi relatada por Issa (1983), Pinto (1997) e também corrobora com os relatos de Nowell \& Laing (1998), os quais afirmam que os fungicidas do grupo químico do triazol, foram eficientes no controle desse patógeno. Três aplicações de propiconazol ( $\left.1 \mathrm{~L} \mathrm{ha}{ }^{-1}\right)$, foram eficazes na redução da taxa de desenvolvimento da mancha foliar por E. turcicum em milho (Bowen \& Pederson, 1988). 
Gonzalez \& Gonzalez-Varela (2007) relataram, em estudos da concentração mínima inibitória in vitro, que o fungicida azoxistrobina não foi satisfatório para o controle deste patógeno. $\mathrm{Na}$ cultura do sorgo, Cota et al. (2010) relataram que a mistura dos fungicidas epoxiconazol + piraclostrobina $\left(0,75 \mathrm{~L} \mathrm{ha}^{-1}\right)$ foi eficiente no controle de E. turcicum.

Os triazóis apresentam maior sistemicidade, o que confere uma característica de maior mobilidade, ocupando mais rapidamente os sítios infectados ou passíveis de infecção. Ao passo que as estrobilurinas apresentam uma ação sistêmica mais lenta, propriedade que confere maior residual e, portanto, maior eficiência em aplicações preventivas (Hewitt, 1998).

\section{CONCLUSÃO}

Os fungicidas protioconazol + trifloxistrobina, propiconazol e ciproconazol + azoxistrobina apresentam controle satisfatório por até 21 dias após a inoculação de $E$. turcicum.

Para o controle curativo de E. turcicum os fungicidas ciproconazol + azoxistrobina, benzovindiflupir + azoxistrobina, propiconazol, protioconazol + trifloxistrobina e azoxistrobina fungicidas apresentam controle satisfatório até 15 dias após a inoculação

O fungicida protioconazol + trifloxistrobina apresenta a menor taxa de expansão de lesão $\left(0,45 \mathrm{~mm}^{2} \mathrm{dia}^{-1}\right)$ de E. turcicum quando aplicado curativamente.

\section{REFERÊNCIAS}

Abbot, W. S. (1925). A method of computing the efectiveness of na inseticide. Journal Economic Entomology, 18(2), 265-267. Recuperado de https://academic.oup.com/jee/articleabstract/18/2/265/785683/A-Method-of-Computing-the-Effectiveness-ofan?redirectedFrom=fulltext. doi: 10.1093/jee/18.2.265.

Alfenas, A. C.; \& Mafia, R. G. (2007). Métodos em Fitopatologia. Viçosa: Editora UFV.

Alvim, K. R.; Brito, C. H.; Gomes, L. S.; Brandão, A. M.; \& Oliveira, F. H. (2010). Severidade e controle da helmintosporiose comum (Exserohilum turcicum) em oito híbridos comerciais em Jataí-GO. XXVIII Congresso Nacional de Milho e Sorgo.

Azevedo, L. A. S. (2007). Fungicidas sistêmicos: teoria e prática. (1a ed.) Campinas: EMOPI.

Boller, W.; Forcelini, C. A.; \& Hoffmann, L. L. (2007). Tecnologia de aplicação de fungicidas - Parte I. In: DA LUZ, W. C. (Ed.). Revisão Anual de Patologia de Plantas, 15. Passo Fundo: Gráfica e Editora Padre Berthier dos Missionários da Sagrada Família.

Bowen, K. L.; \& Pedersen, W. L. (1988). Effects of northern leaf blight and detasseling on yields and yield components of corn inbreds. Plant Disease, St. Paul, 72, 952-956. Recuperado de https://www.apsnet.org/publications/PlantDisease/Backlssues/Documents/1988Articles/Plan tDisease72n11_952.pdf. 
Companhia Nacional de Abastecimento. (2017). Acompanhamento da safra brasileira de grãos, (4) Safra 2016/17 - Décimo segundo levantamento, Brasília. Recuperado de http://www.conab.gov.br/OlalaCMS/uploads/arquivos/17_09_12_10_14_36_boletim_graos_ setembro_2017.pdf.

Cota, L. V.; Costa, R. V.; Silva, D. D.; \& Parreira, D. F. (2010). Recomendação para o controle químico da helmintosporiose do sorgo (Exserohilum turcicum). Sete Lagoas: Embrapa Milho e Sorgo (Circular técnica, 149).

Elliot, C. H.; \& Jenkins, M. T. (1946). Helminthosporium turcicum leaf blight of corn. Phytopathology, St. Paul, 36, 660.

Esteves, M. C. F. (1989). Reações a Exserohilum turcicum (Pass.) Leonard \& Suggs em milho (Zea mays L.) e variabilidade do patógeno. (Tese de mestrado). Escola Superior de Agricultura "Luiz de Queiróz", Universidade de São Paulo, Piracicaba, SP, Brasil.

Forcelini, C. A,; Goellner, C. I. \& Mio, L. L. M. (2001). Resistência de fungos a fungicidas. Revisão Anual de Patologia de Plantas, Associação, 9, 339-381.

Genet, J. L.; Jaworska, G.; Geddens, R.; \& Sheperd, C. (2000). Effect of temperature on the curative and anti-sporulant action of cymoxanil for control of Phytophthora infestans. Fifth Workshop of an European Network for development of an Integrated Control Strategy of potato late blight, Munich, 107-117.

Ghini, R.; \& Kimati, H. (2000). Resistência de fungos a fungicidas. Jaguariúna: EMBRAPA Meio Ambiente.

Gomes, E. C. S.; Leite, R. P.; Silva, F. J. A.; Cavalcanti, L. S.; Nascimento, L. C.; \& Silva, S. M. (2014). Manejo do míldio e ferrugem em videira com indutores de resistência: produtividade e qualidade pós-colheita. Tropical Plant Pathology, 36(5), 332-335. Recuperado de: http://www.scielo.br/pdf/tpp/v36n5/v36n5a09.pdf. doi: 10.1590/S198256762011000500009.

Gonzalez, A. J.; \& Gonzalez-Varela, G. (2007). Ensayo in vitro de fungicidas frente a Exserohilum turcicum, agente causal del tizón norteño del maíz, en Asturias. Boletín de sanidad vegetal. Plagas, 33, 289-295. Recuperado de http://www.mapama.gob.es/ministerio/pags/biblioteca/revistas/pdf_Plagas/BSVP_33_02_28 9_295.pdf.

Hewitt, H. G. (1998). Fungicides in Crop Protection. Cambridge: CAB Internacional.

Issa, E. (1983). Controle químico de Helminthosporium turcicum Pass. em milho pipoca, Zea mays L. O Biológico, Campinas, 49(2), 41-43.

Lago, F. L.; \& Nunes, J. (2008). Avaliação da produtividade de milho em relação à aplicação de fungicidas em diferentes estádios. Cultivando O saber, Cascavel, 1, 17-23. 
Linhares, A. I.; \& Ghini, R. (2001). Resistência de fungos fitopatogênicos inibidores de metilação (DMI): uma revisão. Jaguariúna: Embrapa Meio Ambiente.

Nowell, D. C.; \& Laing, M. D. (1998). Evaluation of fungicides to control Exserohilum turcicum on sweet corn in South Africa. Journal of the Southern Africa Society for Horticultural Sciences, Stellenbosch, 8(2), 65-69. Recuperado de http://agris.fao.org/agrissearch/search.do?recordID=ZA1999000206.

Pinto, N. F. J. A. (2004). Controle químico de doenças foliares em milho. Revista Brasileira de Milho e Sorgo, Sete Lagoas, 3(1), 134-138. Recuperado de http://rbms.cnpms.embrapa.br/index.php/ojs/article/viewFile/96/97.

Pinto, N. F. J. A.; Angelis, B.; \& Habe, M. H. (2004). Avaliação da eficiência de fungicidas no controle da Cercosporiose (Cercospora zeae-maydis) na cultura do milho. Revista Brasileira de Milho e Sorgo, Sete Lagoas, 3(1), 139- 145.

Pinto, N. F. J. A. (1997). Eficiência de fungicidas no controle de doenças foliares de milho, Summa Phytopathologica, 23, 271-274. Recuperado de https://ainfo.cnptia.embrapa.br/digital/bitstream/item/66398/1/Eficiencia-fungicidas-1.pdf.

Raymundo, A. D.; \& Hooker, A. L. (1981). Measurind the relationship between northern corn blight and yield losses. Plant Diseases. St. Paul, 65, 325-327.

Reis, E. M.; Reis, A. C.; \& Carmona, M. A. (2010). Manual de fungicidas: guia para controle químico de doenças de plantas. (6a ed.). Passo Fundo. Ed: Universidade de Passo Fundo.

United States Department of Agriculture. (2017). USDA Agricultural Projections. Recuperado de http://www.ers.usda.gov/ publications/oce081. 